\title{
Treat-to-target (T2T) of serum urate (SUA) in gout: a clinical audit in real-world gout patients
}

\author{
C.L. Teh'1, Y.K. Cheong', S.A. Wan', G.R. Ling ${ }^{2}$ \\ ${ }^{1} R$ heumatology Unit, Department of Medicine, Sarawak General Hospital, Sarawak, Malaysia; \\ ${ }^{2}$ Rheumatology Unit, Department of Medicine, Sibu Hospital, Sarawak, Malaysia
}

\begin{abstract}
SUMMARY
Treat-to-target (T2T) for gout has been established recently to improve its management, which has been reported to be sub-optimal with significant gaps between the goals of treatment and day-to-day clinical practice. T2T recommended a goal of serum urate (SUA) target of $<360 \mu \mathrm{moI} / \mathrm{L}$ in all patients with gout and $<300 \mu \mathrm{moI} / \mathrm{L}$ in patients with tophaceous or severe gout. T2T strategy was applied in the management of gout patients in two Rheumatology clinics from 1 January 2016 onwards. We performed a clinical audit to assess T2T of SUA in gout patients and to identify causes for failure to achieve target SUA among them.

There were a total of 304 patients for our analysis. They were of multi-ethnic origin with male predominance $(88.8 \%)$. They had a mean age of $57.7+13.7$ years and mean disease duration of $10.1+8.7$ years. The most common comorbidities were hypertension (76.2\%), dyslipidemia (52.5\%) and diabetes mellitus (DM) (27.4\%). Our patients' body mass indexes showed that $47.7 \%$ were obese while $34.2 \%$ were overweight. Up to $62.4 \%$ of our patients had tophi and $42.6 \%$ had joint deformities. Only $34.9 \%$ of patients achieved target SUA. Nonadherence $(52.3 \%)$ was the main reason identified for failure to achieve target SUA. The independent predictors for failure to achieve target SUA were nonadherence $(\mathrm{HR}=7.84, \mathrm{p}=0.000)$ and presence of tophi $(\mathrm{HR}=1.95, \mathrm{p}=0.001)$.
\end{abstract}

Key words: Treat to target (T2T); gout.

Reumatismo, 2019; 71 (3): 154-159

\section{INTRODUCTION}

out is the most common inflammatory arthritis worldwide. The prevalence and incidence of gout is increasing (1) due to global aging, dietary and lifestyle changes. There is a strong association between gout and other metabolic disorders such as hypertension, dyslipidemia and DM (2-4). Gout is also an independent risk factor for cardiovascular morbidity and mortality.

Treat-to-target (T2T) guidelines for gout have been published in recent years to guide clinicians in managing gout patients. These treatment guidelines (5-7) support a treat-to-target strategy in gout with a goal of SUA target of $<360 \mu \mathrm{moI} / \mathrm{L}$ in all patients with gout and $<300 \mu \mathrm{mol} / \mathrm{L}$ in those with tophaceous or severe gout. Evidence suggest failure to achieve treatment goals in gout is associated with worsening outcomes such as recurrent flares, tophi and joint deformities. Gout is often misdiagnosed and inadequately treated with significant gaps between the goals of treatment and actual clinical practice (8-11).

We performed a clinical audit to assess T2T of SUA in gout patients and to investigate the causes of failure to achieve target SUA among our patients.

\section{MATERIALS AND METHODS}

T2T strategy was applied in of all our patients with gout who were managed in two rheumatology clinics in Sarawak from 1 Jan 2016 onwards. A retrospective case notes review was carried out from $1^{\text {st }}$ June 2017 through $31^{\text {st }}$ December 2017 to assess T2T of SUA.

All patients who were diagnosed with gout according to 1977 American Rheumatism Association preliminary criteria for the classification of the acute arthritis of pri- 
mary gout (12) were included in the study. Demographics of patients, date of gout onset, duration of disease and comorbidities (including hypertension, DM, dyslipidemia and stroke) were collected from patient case notes. We also evaluated clinical presentation of gout, treatment regimes (for both acute and chronic gout) and laboratory investigations (including SUA at first visit and at last follow-up, glucose level and lipids profile). Renal function was measured using serum creatinine which was available from the patient records and classified according to the KDIGO 2012 Clinical Practice Guideline for the Evaluation and Management of Chronic Kidney Disease (13). Nonadherence was defined as documentation of any non-compliance with medical therapy in the medical records.

The major outcome was achievement of SUA of $<360 \mu \mathrm{moI} / \mathrm{L}$ in all patients with gout and $<300 \mu \mathrm{moI} / \mathrm{L}$ in those with tophaceous or severe gout. The major outcome was determined based on the latest serum urate level of the patients. Data was collected till patients' last follow up during the study period.

Analysis was performed using SPSS version 10.0 (SPSS, Chicago IL, USA). Results are presented as frequencies and percentages for categorical variables and as means and standard deviations (SD) for continuous variables. The risk factors associated with failure to achieve SUA were assessed using multivariate Cox regression analysis.

\section{RESULTS}

\section{Patients' characteristics}

There were a total of 304 patients available for our analysis. Our patients were of multi-ethnic origins with a male predominance (271 males versus 33 females). They had a mean age of $57.7 \pm 13.7$ years and a mean disease duration of $10.1 \pm 8.7$ years as illustrated in Table I. The most common comorbidities in our patients were hypertension $(76.2 \%)$, dyslipidemia $(52.5 \%)$ and DM (27.4\%). Ischemic heart disease was found in $10.6 \%$ of patients while $7.3 \%$ had a stroke. In this current study, $36 \%$ of pa- tients had more than two comorbidities. Grade 2 Chronic Kidney Disease (CKD) was present in $41.9 \%$ of patients while Grade 3 CKD was present in $32.7 \%$. Grade 4 CKD was present in $8.9 \%$ of patients and $3.6 \%$ of patients had End Stage Renal Failure.

Body mass indexes were available in $73.6 \%$ of patients in which $48 \%$ were obese while $35 \%$ were overweight. Most of our patients were smokers $(73.4 \%)$. Alcohol consumption was reported among $20.1 \%$ of patients. Positive family history of gout was found in $33.9 \%$ of patients.

\section{Pattern of gout}

Up to $62.4 \%$ of our patients had tophi and $42.6 \%$ had joint deformities. Only 9.9\%

Table I - Demographic characteristics of patients $(n=304)$ (values are mean $\pm S D$ ).

\begin{tabular}{|c|c|}
\hline Age (years) & $57.7+13.7$ \\
\hline Sex (male/female) & $271 / 33(11.2 \% / 88.8 \%)$ \\
\hline Ethnicity -Iban & $125(41.0 \%)$ \\
\hline Malay & $83(27.0 \%)$ \\
\hline Chinese & $54(18.0 \%)$ \\
\hline Bidayuh & $26(9.0 \%)$ \\
\hline Others & $16(5.0 \%)$ \\
\hline Duration of disease (years) & $10.1+8.7$ years \\
\hline $\begin{array}{l}\text { Comorbidities - } \\
\text { Hypertension }\end{array}$ & $231(76.2 \%)$ \\
\hline Dyslipidemia & $159(52.5 \%)$ \\
\hline DM & $83(27.4 \%)$ \\
\hline Chronic renal failure & $72(23.8 \%)$ \\
\hline Ischemic heart disease & $32(10.6 \%)$ \\
\hline Stroke & $22(7.3 \%)$ \\
\hline End stage renal failure & $11(3.6 \%)$ \\
\hline BMI - underweight & $5(1.7 \%)$ \\
\hline Normal & $33(10.9 \%)$ \\
\hline Overweight & $78(25.7 \%)$ \\
\hline Obese & $107(35.3 \%)$ \\
\hline Unavailable & $80(26.4 \%)$ \\
\hline Tophi & $189(62.4 \%)$ \\
\hline Joint deformities & $129(42.6 \%)$ \\
\hline Colchicine & $228(75.0 \%)$ \\
\hline Allopurinol & $266(87.8 \%)$ \\
\hline Probenecid & $4(1.3 \%)$ \\
\hline Febuxostat & $5(1.7 \%)$ \\
\hline Allopurinol and probenecid & $4(1.3 \%)$ \\
\hline Febuxostat and probenecid & $1(0.3 \%)$ \\
\hline
\end{tabular}


of patients had history of uric acid stones and only $9.2 \%$ had history of uric acid nephropathy. Patients with tophi were found to have a longer disease duration than those without tophi $(11.6 \pm 8.8$ years vs $7.6 \pm 8.1$ years, $\mathrm{p}=0.000$ ). They were more likely to consume alcohol compared to those without tophi $(\mathrm{p}=0.008)$. There were no differences in prevalence of hypertension, dyslipidemia and chronic kidney disease between these two groups of patients. The common medications received by patients included aspirin $(17.5 \%)$, diuretics $(10.2 \%)$ and pyrazinamide (3.3\%).

Most of our patients presented with acute gouty arthritis (59.5\%). Mono arthritis (90\%) was the main presentation in acute gout attack. Colchicine $(80.2 \%)$ was the main medication given to treat the acute gout attacks. Systemic steroids $(49.5 \%)$ and tramadol/codeine $(46.2 \%)$ were the other two main medications utilized to treat acute gout. NSAIDS was only used in $13.7 \%$ and intraarticular steroids were used in $10.4 \%$.

Colchicine prophylaxis was given in $75 \%$ of patients for long-term management of gout. Urate-lowering therapies (ULT) were given to $280(92.4 \%)$ patients, of which allopurinol was the main therapeutic agent (87.8\%). Febuxostat was given to $1.7 \%$ of patients. Similarly, probenecid was also given to only $1.3 \%$ of patients. Combination therapy of allopurinol and probenecid was given to $1.3 \%$ patients while $0.3 \%$ patients were receiving combination therapies of febuxostat and probenecid. Hypersensitivity to allopurinol was detected in $4.3 \%$ of patients. Titration of allopurinol was done in $83.1 \%$ of patients who received the drug.

Our patients had a baseline SUA of $599.9+137.0$ umol/L. Their SUA levels were at a mean of $415.59 \pm 142.29 \mathrm{umol} / \mathrm{L}$ at last follow up.

Patient education (which comprised of one-to-one counselling by the doctors) was administered to all patients in the study at every clinic visit. Screening for comorbidities during the past 12 months was carried out in the patients as followed: hypertension $(98.7 \%)$, dyslipidemia $(67.3 \%)$ and DM $(62.2 \%)$. Serum creatinine was performed in $94 \%$ of patients to screen for renal impairment. All patients had regular urate levels assessment every 3 to 6 months during the study period.

\section{Treat-to-target of serum urate}

Only $34.9 \%$ of patients achieved target SUA as illustrated in Table II. The mean time to achieving target was $28.5 \pm 43.8$ months among the patients who achieved treatment targets. Patients who achieved treatment targets had a longer mean disease duration $(12.3 \pm 9.7$ years $v s 8.9 \pm 7.9$ years, $\mathrm{p}=0.002$ ) and were treated longer compared to those who did not achieve target SUA (47.4+36.8 months vs $25.1 \pm 25.9$ months, $\mathrm{p}=0.000$ ). Patients who achieved target

Table II - Characteristics of gout patients who achieved target SUA versus those who failed to achieve target SUA.

\begin{tabular}{|l|c|c|c|}
\hline & Achieved target SUA & Failure to achieve target SUA & p-value \\
\hline Numbers(n) & 106 & 198 & - \\
\hline Age (years) & $58.2+13.2$ & $57.4+14.0$ & 0.623 \\
\hline Disease duration (years) & $12.3+9.7$ & $8.9+7.9$ & 0.002 \\
\hline Treatment duration(months) & $47.4+36.8$ & $25.1+25.9$ & 0.000 \\
\hline Hypertension & 86 & 145 & 0.142 \\
\hline Dyslipidemia & 61 & 98 & 0.195 \\
\hline DM & 29 & 54 & 0.992 \\
\hline CKD & 40 & 97 & 0.055 \\
\hline Ischemic heart disease & 7 & 25 & 0.100 \\
\hline Tophi & 47 & 14 & 0.008 \\
\hline Nonadherence & 4 & 87 & 0.000 \\
\hline Allopurinol dose (mg/day) & $370.6+177.0$ & $308.3+236.5$ & 0.015 \\
\hline
\end{tabular}


Table III - Univariate analysis of the risk factors for failure to achieve target SUA.

\begin{tabular}{|l|c|c|c|c|}
\hline & & \multicolumn{3}{|c|}{ 95\% Confidence interval } \\
\hline & Hazard ratio & Lower & Upper & p-value \\
\hline Age & 0.994 & 0.979 & 1.009 & 0.425 \\
\hline Gender & 1.006 & 0.736 & 1.376 & 0.968 \\
\hline Ethnic group & 0.950 & 0.816 & 1.105 & 0.502 \\
\hline CKD & 1.231 & 0.830 & 1.825 & 0.301 \\
\hline Hypertension & 1.287 & 0.788 & 2.101 & 0.313 \\
\hline Duration of treatment & 1.003 & 0.998 & 1.009 & 0.252 \\
\hline Obesity & 1.060 & 0.689 & 1.629 & 0.791 \\
\hline
\end{tabular}

Table IV - Multivariate analysis of the risk factors for failure to achieve target SUA.

\begin{tabular}{|l|c|c|c|c|}
\hline & & \multicolumn{3}{|c|}{ 95\% Confidence interval } \\
\hline & Hazard ratio & Lower & Upper & p-value \\
\hline Nonadherence & 7.849 & 2.888 & 21.334 & 0.000 \\
\hline Presence of Tophi & 1.953 & 1.322 & 2.885 & 0.001 \\
\hline
\end{tabular}

SUA were on higher mean daily allopurinol doses $(370.6 \pm 177.0 \mathrm{mg}$ vs $308.3 \pm 236.5$ $\mathrm{mg}, \mathrm{p}=0.015$ ). For those who achieved target SUA, patients with tophi were on higher doses of allopurinol compared to those without tophi $(417.4 \pm 188.6 \mathrm{mg}$ vs $306.0 \pm 137.1$, $\mathrm{p}=0.006$ ). Up to $40.8 \%$ of patients were still experiencing gout attacks, with a frequency of $6.8 \pm 8.2$ times per year.

Nonadherence $(52.3 \%)$ was the main reason identified for failure to achieve target SUA followed by the presence of renal dysfunction (23.6\%), inadequate dose titration of ULT (13.2\%) and inefficacy of ULT $(6.3 \%)$. Intolerance to ULT was the cause of treatment failure in $4.6 \%$ of patients.

The independent predictors for failure to achieve target SUA were nonadherence $(\mathrm{HR}=7.85, \mathrm{p}=0.000)$ and presence of tophi $(\mathrm{HR}=1.95, \mathrm{p}=0.001)$ (Tables III and IV). Age, gender, ethnicity, presence of comorbidities and presence of CKD were not associated with failure to achieve target SUA. Alcohol consumption was associated with nonadherence to treatment $(\mathrm{HR}=2.36$, $\mathrm{p}=0.000$ ). Age, number and types of comorbidities as well as presence of tophi were not associated with nonadherence.

\section{DISCUSSION}

Our study showed that only $34.9 \%$ of gout patients achieved the target SUA recommended by T2T; this figure is significantly lower than that reported in studies in which up to $80 \%$ of patients were able to achieve the treatment goal (14-16). This is most probably due to the fact that our patients were complicated gout patients with tophi and erosions. The majority of our patients had chronic tophaceous gout with a significant burden of hypertension, dyslipidemia, DM and obesity. In this, they were similar to the reported rates of metabolic disorders from other studies worldwide (2-4). Our patients also had a high incidence of chronic kidney disease which limited the optimal use of ULT in achieving target serum urate levels.

Patients who achieved target SUA had a longer disease duration and were treated longer than those who failed to achieve target SUA. They were also on higher mean daily allopurinol doses. This finding shows that achieving target SUA is feasible with a longer treatment period and a higher allopurinol dose. Barriers to achieving target SUA include patient factors and physician factors. Poor understanding of the disease, stigmatization of gout and nonadherence to treatment were the main patient factors identified in failure to achieve target SUA. Physician factors included knowledge gaps, including specialized knowledge of the disease, and clinical inertia in implementing guidelines.

Nonadherence was the main reason for failure to achieve target SUA in this current 
study. Adherence to ULT in gout has been reported as ranging from $18 \%-44 \%$; one of the lowest among patients with chronic diseases (17-21). Nonadherence has been reported as a common cause for ULT failure $(20,22)$. Rashid et al. showed that adherent patients were 2.5 -fold more likely than nonadherent patients to achieve an SUA $<6.0$ $\mathrm{mg} / \mathrm{dL}$ in their study (23). Younger age, fewer comorbidities or gout complications, race, and prescriber type predicted poor adherence to $\operatorname{ULT}(24,25)$. One recent meta analysis showed that older age, more comorbidities, and the presence of DM or hypertension were associated with medication adherence among patients with gout (21). Concomitant diuretic use, prescriber specialty, and allopurinol dosing practices had been identified as potentially modifiable factors associated with treatment adherence and achieving treatment target (23). Use of nonsteroidal anti-inflammatory drugs (NSAIDs) and systemic glucocorticoids reduced resumption of allopurinol therapy after a period of non-adherence (22). We found that alcohol consumption was associated with nonadherence among our patients. Alcohol use has been reported to be associated with nonadherence in other medical conditions $(26,27)$. In contrast, age of patients, number and type of comorbidities, tophi and renal failure were not associated with nonadherence in this current study. Nonadherence to ULT remained the major hurdle in the management of gout patients. Patient education that focuses on pharmacologic therapy and lifestyle modifications with medication titration has been found to improve adherence, reduce the number of attacks, and improve the number of patients who achieved T2T of SUA (14-16, 28). Optimization of control of SUA is achievable, even in the setting of renal impairment, by redesigning and implementing processes involving changes in physicians' prescribing habits, enhanced nursing interventions, and patient empowerment and education (14).

There are a few limitations to this study. Firstly, there was selection bias as only gout patients undergoing follow-up at rheumatology departments were included in this study. Our cohort had severe or complex gout. Secondly, we did not collect any patient reported outcomes. Nevertheless, our study reported experience of real-life data of gout patients treated in daily clinical practice.

In summary, we found that the majority of our gout patients failed to achieve target SUA. Nonadherence was the main cause of failure to achieve SUA. Nonadherence and presence of tophi were independent prognostic predictors of failure to achieve SUA. Alcohol consumption was associated with nonadherence to treatment.

Conflict of interest statement: the authors declared no potential conflicts of interest with respect to the research, authorship, and/or publication of this article. The results presented in this paper have not been published previously in whole or part, except in abstract format.

Contributions: all authors were involved in conception or design, or analysis and interpretation of data, or both. All authors approved the final version of the manuscript.

Funding: the authors received no financial support for the research, authorship, and/or publication of this article.

Acknowledgments: the authors would like to thank the Director General of Health, Malaysia for technical supports for this research paper.

\section{REFERENCES}

1. Kuo CF, Grainge MJ, Zhang W, Doherty P. Global epidemiologyof gout: prevalence, incidence and risk factors. Nat Rev Rheumatol. 2015; 11: 649-62.

2. Ragab G, Elshahaly M, Bardin T. Gout: An old disease in new perspective - Areview. J Adv Res. 2017; 8: 495-511.

3. Primatesta P, Plana E, Rothenbacher D. Gout treatment and comorbidities: a retrospective cohort study in a large US managed care population. BMC Musculoskelet Disord. 2011; 12: 103.

4. Zhu Y, Pandya BJ, Choi HK. Comorbidities of gout and hyperuricemia in the US general population: NHANES 2007-2008. Am J Med. 2012; 125: 679-87.e671. 
5. Kiltz U, Smolen J, Bardin T, et al. Treat-totarget (T2T) recommendations for gout. Ann Rheum Dis. 2017; 76: 632-8.

6. Richette P, Doherty M, Pascual E, et al. 2016 updated EULAR evidence-based recommendations for the management of gout. Ann Rheum Dis. 2016; 76: 29-42.

7. Khanna D, Fitzgerald JD, Khanna PP, et al. 2012 American College of Rheumatology guidelines for management of gout. Part 1: systematic nonpharmacologic and pharmacologic therapeutic approaches to hyperuricemia. Arthritis Care R. 2012; 64: 1431-46.

8. Cottrell E, Crabtree V, Edwards JJ, Roddy E. Improvement in the management of gout is vital and overdue: an audit from a UK primary care medical practice. BMC Fam Pract. 2013; 14: 170

9. Arroll B, Bennett M, Dalbeth N, et al. More allopurinol is needed to get gout patients $<0.36 \mathrm{mmol} / \mathrm{L}$ : a gout audit in the form of a before-after trial. J Prim Health Care. 2009; 1: 315-8

10. Wall GC, Koenigsfeld CF, Hegge KA, Bottenberg MM. Adherence to treatment guidelines in two primary care populations with gout. Rheumatol Int. 2010; 30: 749-53.

11. Roddy E, Zhang W, Doherty M. Concordance of the management of chronic gout in a UK primary-care population with the EULAR gout recommendations. Ann Rheum Dis. 2007; 66: 1311-5.

12. Wallace SL, Robinson H, Masi AT, et al. Preliminary criteria for the classification of the acute arthritis of primary gout. Arthritis Rheum. 1997; 20: 895-90.

13. KDIGO 2012 Clinical Practice Guideline for the Evaluation and Management of Chronic Kidney Disease. Kidney Int Suppl. 2013; 3: 19-62.

14. Lim AY, Shen L, Tan CH, et al. Achieving treat to targetin gout: a clinical practice improvement project. Scand J Rheumatol. 2012; 41: 450-7.

15. Janssen CA, Jansen TLTA, Oude Voshaar $\mathrm{MAH}$, et al. Quality of care in gout: a clinical audit on treating to the target with urate lowering therapy in real-world gout patients. Rheumatol Int. 2017; 37: 1435-440.

16. Corbett EJM, Pentony P, McGill NW. Achieving serum urate targets in gout: an audit in a gout-oriented rheumatology practice. Int $\mathrm{J}$ Rheum Dis. 2017; 20: 894-7.
17. Yin R, Li L, Zhang G, et al. Rate of adherence to urate-lowering therapy among patients with gout: a systematic review and meta-analysis. BMJ Open. 2018; 8: e017542.

18. De Vera MA, Marcotte G, Rai S, et al. Medication adherence in gout: a systematic review. Arthritis Care Res (Hoboken). 2014; 66: 1551-9.

19. Harrold LR, Andrade SE, Brieasacher BA, et al. Adherence with urate-lowering therapies for the treatment of gout. Arthritis Res Ther. 2009; 11: R46.

20. Richette P, Flipo RN, Patrikos DK. Characteristics and management of gout patients in Europe: data from a large cohort of patients. Eur Rev Med Pharmacol Sci. 2015; 19: 630-9.

21. Scheepers LEJM, van Onna M, Stehouwer CDA, et al. Medication adherence among patients with gout: A systematic review and meta-analysis. Semin Arthritis Rheum. 2018; 47: 689-702.

22. Harrold LR, Andrade SE, Briesacher B, et al. The dynamics of chronic gout treatment: medication gaps and return to therapy. Am J Med. 2010; 123: 54-9.

23. Rashid N, Coburn BW, Wu YL, et al. Modifiable factors associated with allopurinol adherence and outcomes among patients with gout in an integrated healthcare system. J Rheumatol. 2015; 42: 504-12.

24. Solomon DH, Avorn J, Levin R, Brookhart MA. Uric acid loweringtherapy: prescribing patterns in a large cohort of older adults. Ann Rheum Dis. 2008; 67: 609-13.

25. Sheng F, Fang W, Zhang B, et al. Adherence to gout management recommendations of Chinese patients. Medicine (Baltimore). 2017; 96: e8532

26. Halava H, Korhonen MJ, Huupponen R, et al. Lifestyle factors as predictors of nonadherence to statin therapy among patients with and without cardiovascular comorbidities. CMAJ. 2014; 186: E449-56.

27. Braithwaite RS, Mc Ginnis KA, Conigliaro J, et al. A temporal and dose-response association between alcohol consumption and medication adherence among veterans in care. Alcohol Clin Exp Res. 2005; 29: 1190-7.

28. Rees F, Jenkins W, Doherty M. Patients with gout adhere to curative treatment if informed appropriately: proof-of-concept observational study. Ann Rheum Dis. 2013; 72: 826-30. 